\title{
INVENTARIO MICOBIÓTICO DE LA REGIÓN DE TAPALPA, JALISCO, MÉXICO
}

\author{
Etelvina Gándara ${ }^{1}$, Laura GuZmán-Dávalos ${ }^{2,3}$, Gastón Guzmán ${ }^{1}$ \\ y Olivia RodríGuez ${ }^{2}$
}

${ }^{1}$ Instituto de Ecología, A.C., Apdo. postal 63, 91000 Xalapa, Veracruz, México. ${ }^{2}$ Universidad de Guadalajara, Instituto de Botánica, Departamento de Botánica y Zoología, Apdo. postal 1-139, 45101 Zapopan, Jalisco, México. ${ }_{3}^{3}$ Autor para la correspondencia: lguzman@cucba.udg.mx

\section{RESUMEN}

Se presentan 233 taxa (especies y variedades) de hongos (229 macromicetes y cuatro micromicetes) de la región de Tapalpa, Jalisco. El material estudiado fue recolectado en los años 2000 al 2003, principalmente en bosques de Pinus y Quercus. Se examinaron además otros ejemplares depositados en el Herbario IBUG provenientes de dicha área. Se registran por primera vez de Jalisco 21 taxa. Doscientos cinco especies (88\%) pertenecen a Basidiomycota, $22(9.5 \%)$ a Ascomycota y $6(2.5 \%)$ a Myxomycota. Éste constituye el primer inventario micológico de la zona.

Palabras clave: Jalisco, macromicetes, micromicetes, registros nuevos.

\section{ABSTRACT}

A list of 233 taxa (species and varieties) of fungi (229 macromycetes and four micromycetes) from Tapalpa region, State of Jalisco (Mexico) is presented. The studied material was collected from 2000 to 2003, mainly in Pinus and Quercus forests. Additional specimens from the IBUG herbarium gathered in this region were also studied. Twenty-one taxa are first records from Jalisco State. In the studied fungi, $205(88 \%)$ species belong to Basidiomycota, $22(9.5 \%)$ to Ascomycota, and $6(2.5 \%)$ to Myxomycota. This is the first mycological inventory of the area.

Key words: Jalisco, macromycetes, micromycetes, new records. 


\section{INTRODUCCIÓN}

Los estudios sobre los macromicetes de Jalisco se remontan a más de 50 años atrás. Mains (1958) citó el primer hongo macroscópico del estado y fue en la década de los 70 cuando Guzmán (1970) en su monografía sobre Scleroderma consideró varias especies de Jalisco, y Guzmán y Pérez-Patraca (1972) algunas de Panaeolus. El primer trabajo exclusivo de los macromicetes de la entidad lo realizaron Guzmán y García-Saucedo (1973). Posteriormente se publicaron inventarios de algunas regiones del estado como la Barranca de Huentitán, La Primavera, San Sebastián del Oeste, Sierra de Manantlán, Sierra de Quila y Volcán de Tequila (Téllez et al., 1988; Vázquez y Guzmán-Dávalos, 1988; Rodríguez et al., 1994, 2002; Fierros y GuzmánDávalos, 1995; Herrera-Fonseca et al., 2002). Además, se han estudiado los géneros Gymnopilus, Pluteus, Psilocybe, Scleroderma y Volvariella, así como hongos liquenizados y mixomicetes (Guzmán-Dávalos y Guzmán, 1985, 1986; Trujillo et al., 1986; Guzmán-Dávalos y Álvarez, 1987; Álvarez y Guzmán-Dávalos, 1988, 1993, 2009; Vázquez et al., 1989; Vázquez y Guzmán-Dávalos, 1991b; Guzmán, 1998; Rodríguez, 1998; Rodríguez y Guzmán-Dávalos, 2000, 2001).

En particular de Tapalpa se conocían 64 taxa, con Panaeolus antillarum como la primera especie que se citó de la región (Guzmán y Pérez-Patraca, 1972). Posteriormente, Manzi (1976) registró 32, Sánchez-Jácome y Guzmán-Dávalos (1997) cuatro y Guzmán-Dávalos et al. (2001) 14. Además, varias se citaron de Tapalpa en trabajos generales de Jalisco o de México, como los de Guzmán (1970, 1983), Guzmán y García-Saucedo (1973), Chacón y Guzmán (1983), Guzmán-Dávalos y Trujillo (1984), Guzmán et al. (1988), Vázquez y Guzmán-Dávalos (1991a), Vargas et al. (1992, 1993), Guzmán-Dávalos y Guzmán (1995), Montoya et al. (1996) y Rodríguez y Guzmán-Dávalos (2000, 2001). Por otro lado, Gómez (2004) estudió aspectos etnomicológicos de los hongos de Tapalpa. El presente trabajo se enfoca hacia un primer inventario micológico de la misma.

\section{MATERIALES Y MÉTODOS}

El municipio de Tapalpa se encuentra en el centro de la zona sur de Jalisco (Fig. 1), en el sistema montañoso denominado Sierra de Tapalpa y forma parte de la provincia fisiográfica Eje Neovolcánico Transversal (Ferrusquía-Villafranca, 1993). La altitud de la zona de estudio varía entre 1200 a $2800 \mathrm{~m}$ y su vegetación está formada en su gran mayoría por bosques de Pinus, Pinus-Quercus y Quercus, y en pequeñas 


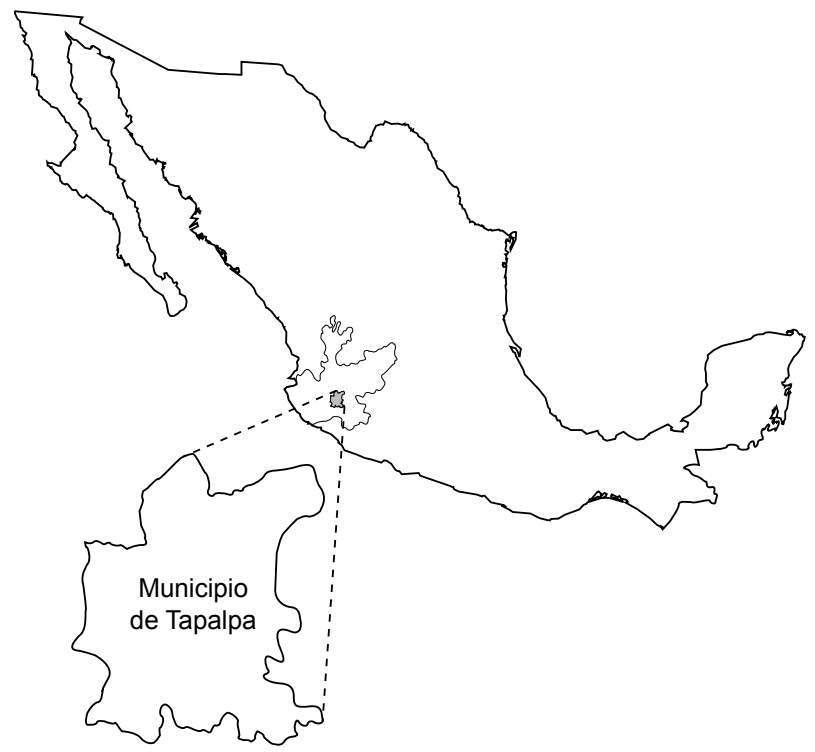

Fig. 1. Ubicación geográfica del municipio de Tapalpa en Jalisco y en México.

áreas por el tropical caducifolio y el mesófilo de montaña (Rzedowski, 1978). Las 11 exploraciones realizadas se concentraron en las tres primeras comunidades vegetales mencionadas, durante los meses de lluvias, de junio a octubre de 2000 al 2003. Desafortunadamente toda la zona estudiada tiene un alto grado de deforestación, debido a intensas prácticas agrícolas, ganaderas y de explotación de madera.

Se recolectaron 789 especímenes que se depositaron en la Colección Micológica del Herbario IBUG. Además se revisaron 360 ejemplares provenientes del municipio de Tapalpa, previamente depositados en IBUG. Las observaciones microscópicas se hicieron a través de cortes con navaja de diferentes partes de los esporomas, montados en soluciones de $\mathrm{KOH}$ o $\mathrm{NH}_{4} \mathrm{OH}$ al 3 o 5\%, rojo Congo, azul de cresil, azul de algodón en lactofenol, reactivo de Melzer y en acetocarmín, según los requerimientos para determinar las especies. Dicha identificación se hizo con base en varias obras, como las de Martin y Alexopoulos (1969), Guzmán (1977), Dennis (1981), Moser (1983), Breitenbach y Kränzlin (1986, 1991) y Gilbertson y Ryvarden (1986, 1987), y en la literatura especializada que se indica en las especies. La información sobre qué hongos se consideran medicinales se tomó de Guzmán (1994, 2008). En el caso de los lignícolas se indicó además cuáles son destructores de la madera, para poder valorar aquéllos con importancia económica. 


\section{RESULTADOS Y DISCUSIÓN}

Especies estudiadas

De los 1149 especímenes de hongos (789 recolectados ex profeso para este trabajo y 360 del Herbario IBUG) se determinaron 578, que corresponden a 233 taxa (Apéndice). El material restante no fue posible estudiarlo por estar mal preservado, falta de datos del esporoma en fresco, o por no contar con literatura especializada en el grupo. De los taxa determinados, 21 (20 especies y una variedad) corresponden a registros nuevos para Jalisco. De los 233 taxa estudiados, 205 (88\%) pertenecen a Basidiomycota, $22(9.5 \%)$ a Ascomycota y $6(2.5 \%)$ a Myxomycota. Esta proporción es del orden de las de listados realizados en otras regiones de Jalisco (Guzmán-Dávalos y Guzmán, 1979; Vázquez y Guzmán-Dávalos, 1988; Rodríguez et al., 1994; Herrera-Fonseca et al., 2002).

Los Basidiomycota estudiados se adscriben a 18 órdenes, de los cuales el de los Agaricales es el mejor representado con 101 taxa (43\%); le siguen Poriales con 41 (17.6\%) y Stereales con 13 (5.6\%). De los Agaricales se estudiaron 15 familias y de ellas las más diversas fueron Tricholomataceae con 21 taxa (20.7\%), Amanitaceae con 19 (18.8\%), Boletaceae con 15 (14.8\%), Russulaceae con 10 (9.9\%), y Agaricaceae, Coprinaceae y Strophariaceae con 7 (6.9\%), cada una. Del orden Poriales la familia más representativa fue Coriolaceae con 28 especies (68\%). Los micromicetes considerados son Apiocrea hyalina, Hypomyces lactifluorum (Fig. 16) y Sepedonium chrysospermum, los cuales se incluyeron porque se habían recolectado con los hongos que parasitan y Cronartium conigenum por ser un parásito común de conos de pinos.

Los 21 taxa que corresponden a primeros registros para Jalisco se indican en el Apéndice con un asterisco. Éstos pertenecen a los Agaricales (14 taxa), Poriales (2), Lycoperdales (1), Phallales (1), Sclerodermatales (1), Stereales (1) y Uredinales (1). Del género Amanita se encontraron tres novedades, entre ellas A. austrostraminea, que se caracteriza por presentar basidioma blanco, píleo con escamas piramidales y pulverulentas, células marginales de la lámina de 20-45(-80) x 12-25 $\mu \mathrm{m}$ y por carecer de fíbulas (Figs. 2-7), lo que coincide con Bas (1969). Amanita tullossii, del complejo de $A$. caesarea (Guzmán y Ramírez-Guillén, 2001), se distingue por tener el subhimenio con (3-)4-5 células de grosor, píleo umbonado y basidiosporas de (7-)9-10(-11) x (5.5-)6-7(-8) $\mu \mathrm{m}$ (Fig. 8). Clitocybe hydrogramma tiene como carácter diagnóstico la presencia de vesículas entre las hifas del pileipellis (Bigelow, 1982) (Figs. 9-10). Hypholoma subviride tiene basidiomas pequeños, con píleo de 9-19 mm de diámetro, ligeramente umbonado, amarillo-anaranjado en el centro a amarillo-limón hacia el margen; sus basidiosporas son de 6-8 x 4-4.5 $\mu \mathrm{m}$, oblon- 
Gándara et al.: Inventario micobiótico de Tapalpa, Jalisco

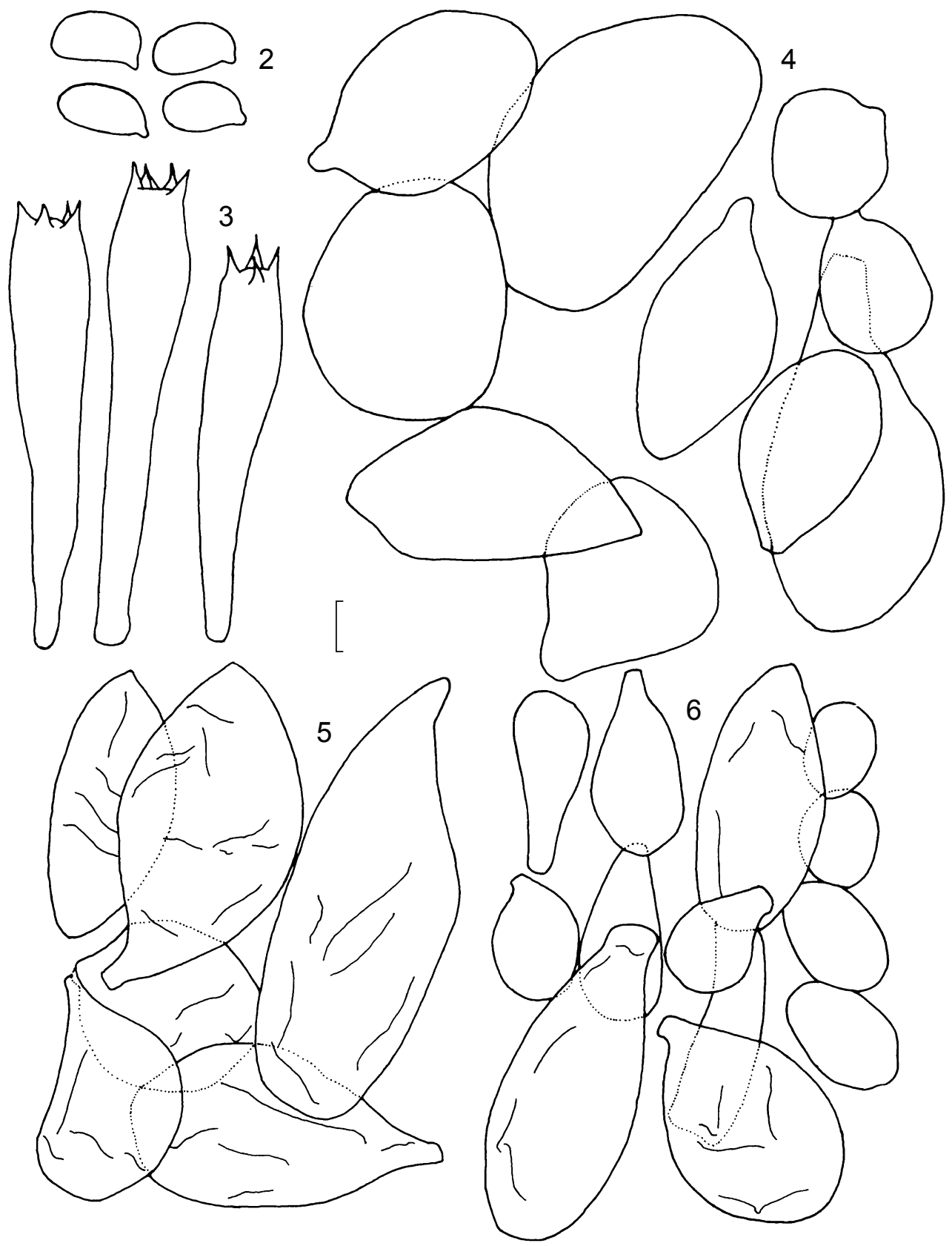

Figs. 2-6: Amanita austrostraminea (Gándara 185). 2. basidiosporas; 3. basidios; 4. células marginales de la lámina; 5 . células del velo sobre la base del estípite; 6 . células del velo sobre el píleo (escamas). Escala figs. 2-3 = $8 \mathrm{~mm}, 4-6=11 \mathrm{~mm}$. 

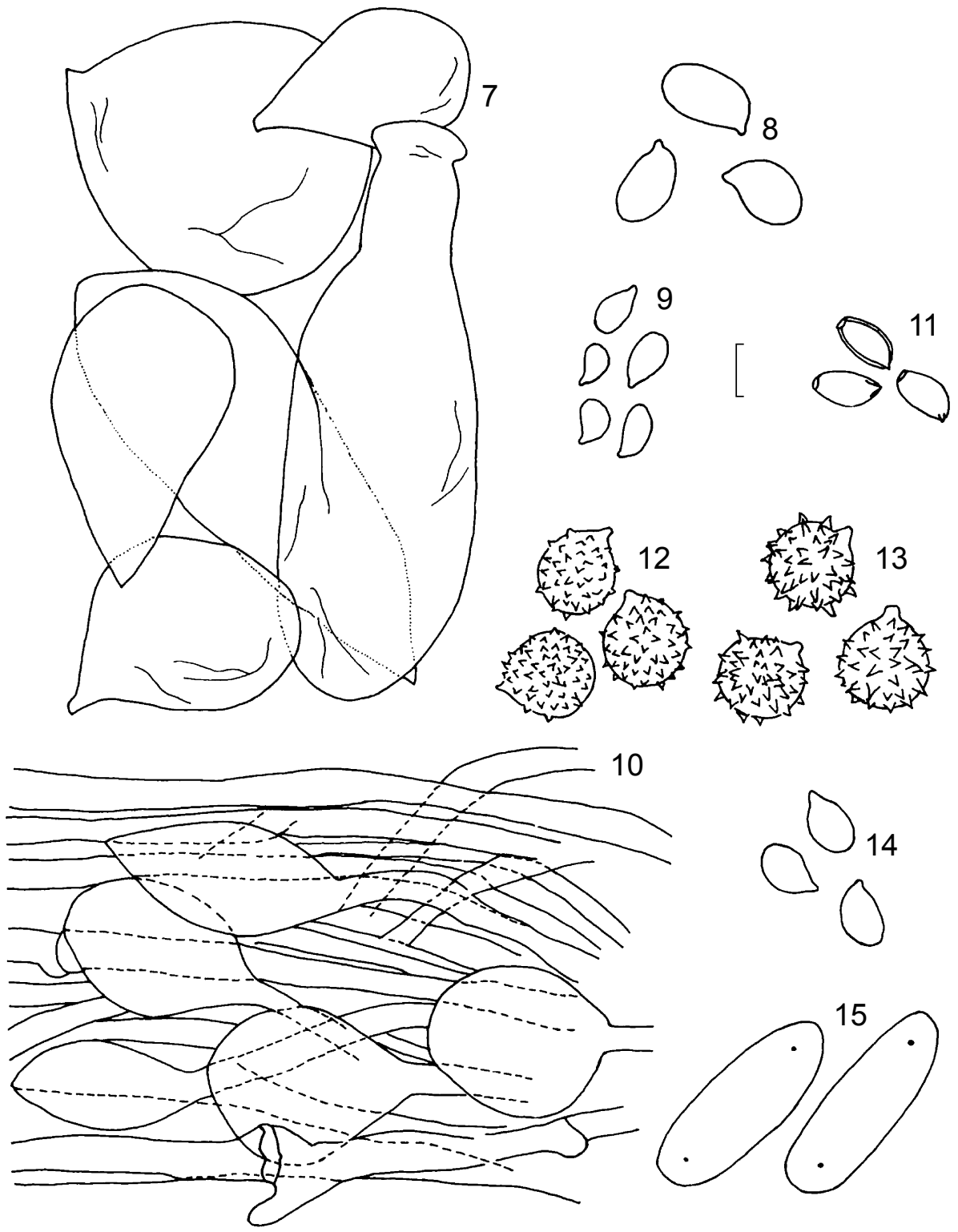

Figs. 7-15. 7. Amanita austrostraminea (Gándara 185), células del anillo. 8. A. tullossii (Gándara 309), basidiosporas. 9-10. Clitocybe hydrogramma (Gómez 86-B): 9. basidiosporas, 10. pileipellis. 11. Hypholoma subviride (Gándara 131), basidiosporas. 12-13. Laccaria ochropurpurea, basidiosporas: 12 (Gándara 119), 13 (Gómez 98). 14. Volvariella cinerea (Gándara 186), basidiosporas. 15: Phallus ravenelii (Gándara 3), basidiosporas. Escala fig. 7 $=11 \mathrm{~mm}$, figs. $8-15=8 \mathrm{~mm}$. 
gas, de pared subgruesa $(0.5 \mu \mathrm{m})$, de color café-amarillento y con poro germinal (Smith, 1951; Pegler, 1977) (Fig. 11). Laccaria ochropurpurea se distingue por su basidioma grande, píleo de 35-100(-125) mm, violáceo a color café-ante y basidiosporas globosas a subglobosas (Mueller, 1992) (Figs. 12-13). Volvariella cinerea tiene píleo de color café con tonos gris-plomo, volva concolora y pubescencia blanca; sus basidiosporas son de 5.5-6.5 x 4.5-5 $\mu \mathrm{m}$, elipsoides, pocas ampliamente elipsoides (Mora, 1985) (Fig. 14). Pachykytospora papyracea presenta basidioma efuso-reflejo, con poros grandes, 2-3 por mm, amarillo-anaranjados (Gilbertson y Ryvarden, 1987). Phallus ravenelii tiene el receptáculo cónico, liso a granuloso y basidiosporas subelipsoides y hialinas (López et al., 1980) (Fig. 15). Gloeoporus thelephoroides se caracteriza por el himenóforo de color rosa pálido a café-rosado y por carecer de fíbulas (Gilbertson y Ryvarden, 1986). Gymnosporangium clavipes es parásito sobre los frutos del tejocote (Crataegus mexicana Moc. \& Sessé), en donde forma ecios de 3-8 mm de longitud (Carrión y Galván, 1984).

Distribución ecológica e importancia de los hongos estudiados

La mayoría de las especies estudiadas provienen de los ecosistemas de clima templado como sigue: 153 taxa se adscriben al bosque de pino-encino y 132 al de pino. Solamente ocho se encontraron en bosque de encino: Albatrellus cristatus (Fig. 24), Amanita rubescens, Ganoderma lobatum, Gymnopus polyphyllus, Lentinula boryana, Omphalotus mexicanus, Phylloporus rhodoxanthus y Stropharia semiglobata; 15 en pastizales, entre las que destacan Agaricus campestris, Calvatia cyathiformis, Chlorophyllum molybdites, Lycoperdon marginatum, Panaeolus antillarum y Psilocybe cubensis. Se observó que algunos bosques de pino-encino de la zona de estudio tienen influencia termófila por su posición topográfica en la vertiente hacia el Océano Pacífico; ello puede incidir en el desarrollo de hongos de afinidades tropicales, como Fistulina guzmanii, Funalia polyzona, Gloeophyllum striatum, Hexagonia hirta, H. tenuis, Lentinus crinitus, Polyporus tenuiculus y Schizophyllum commune. Sin embargo, es curioso que Pycnoporus sanguineus (L.) Murrill, especie de esta vinculación y de amplia distribución en México (Guzmán, 1977), no se encontrara en la zona de estudio.

Referente al sustrato en el que se desarrollan las especies estudiadas (Apéndice), se encontró que 105 (45.1\%) son lignícolas, 96 (41.2\%) terrícolas, 16 (6.9\%) humícolas, 7 (3\%) fimícolas, 4 (1.7\%) fungícolas, 3 (1.3\%) fitopatógenas, y 1 (0.4\%) entomopatógena. De acuerdo con Guzmán-Dávalos y Guzmán (1979), en los bosques de coníferas suele ser mayor el número de especies terrícolas y humícolas, que el de las lignícolas; sin embargo, en el presente trabajo la cantidad de lignícolas (105) es 
cercana a la suma de las terrícolas y humícolas (112). La explicación de esta proporción es posible que se encuentre en la influencia tropical presente en estos bosques o en el fuerte impacto antropogénico que existe en la región, o también al hecho de que no se tomaron en cuenta muchas especies micorrizógenas, como las de Cortinariaceae y Russulaceae, debido a que no fue posible determinar a especie los ejemplares correspondientes en virtud de la complejidad taxonómica de estas familias.

En relación a la importancia de los hongos (Apéndice), 82 (35\%) son comestibles, 81 (35\%) son destructores de madera, 70 (30\%) micorrizógenos, 24 (10.3\%) medicinales, $30(13 \%)$ venenosos, $8(3.4 \%)$ parásitos de hongos, plantas o animales
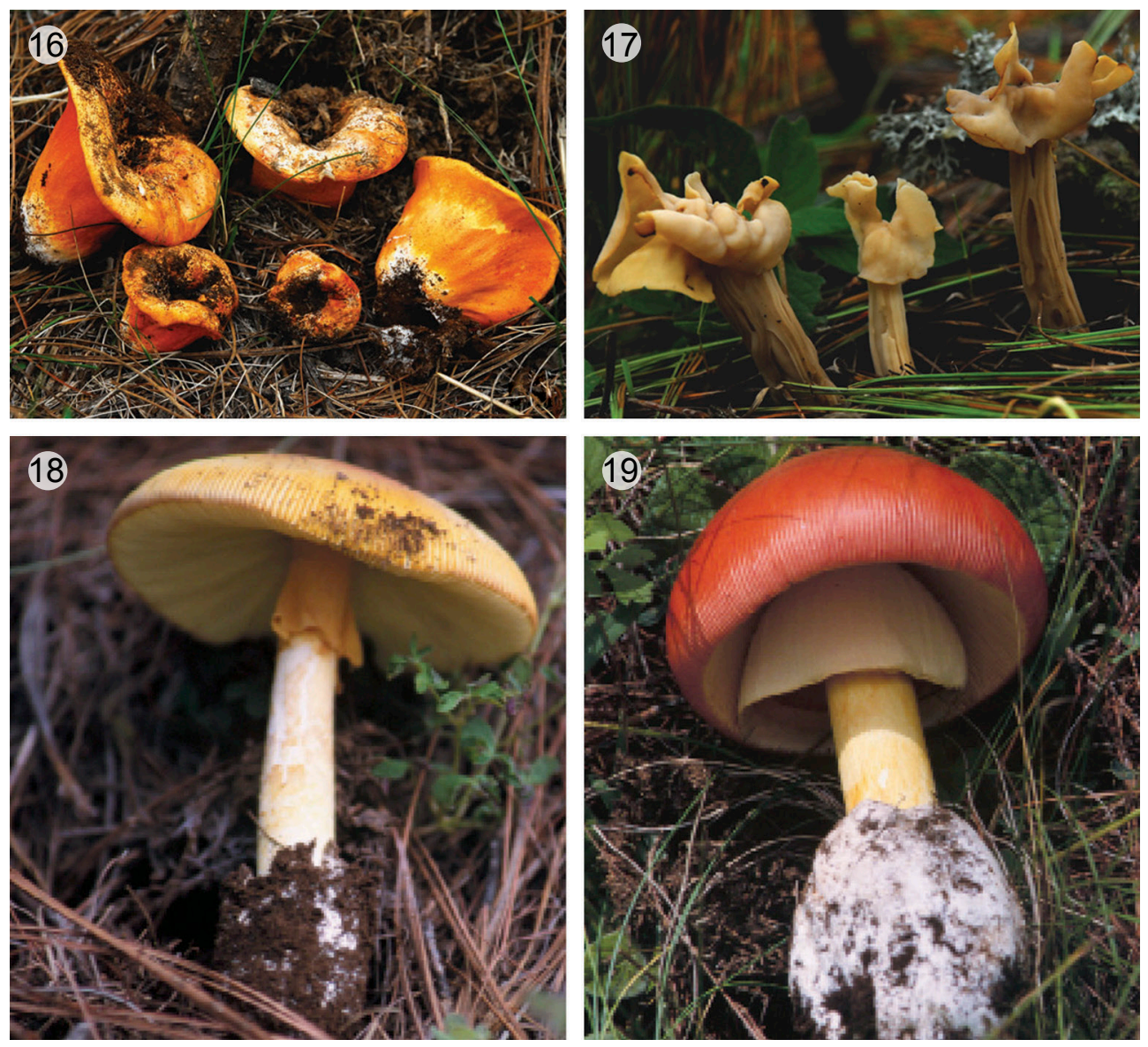

Figs. 16-19. 16. Hypomyces lactifluorum. 17. Helvella crispa. 18. Amanita laurae. 19. A. tecomate. 
y 3 (1.3\%) alucinógenos. Según Gómez (2004), las especies comestibles que se consumen en la región son 12, principalmente: Amanita laurae (Fig. 18), A. tecomate (Fig. 19), A. tullossii (esta última como Amanita sp. en Gómez, 2004) y Lyophyllum decastes (Fig. 20).
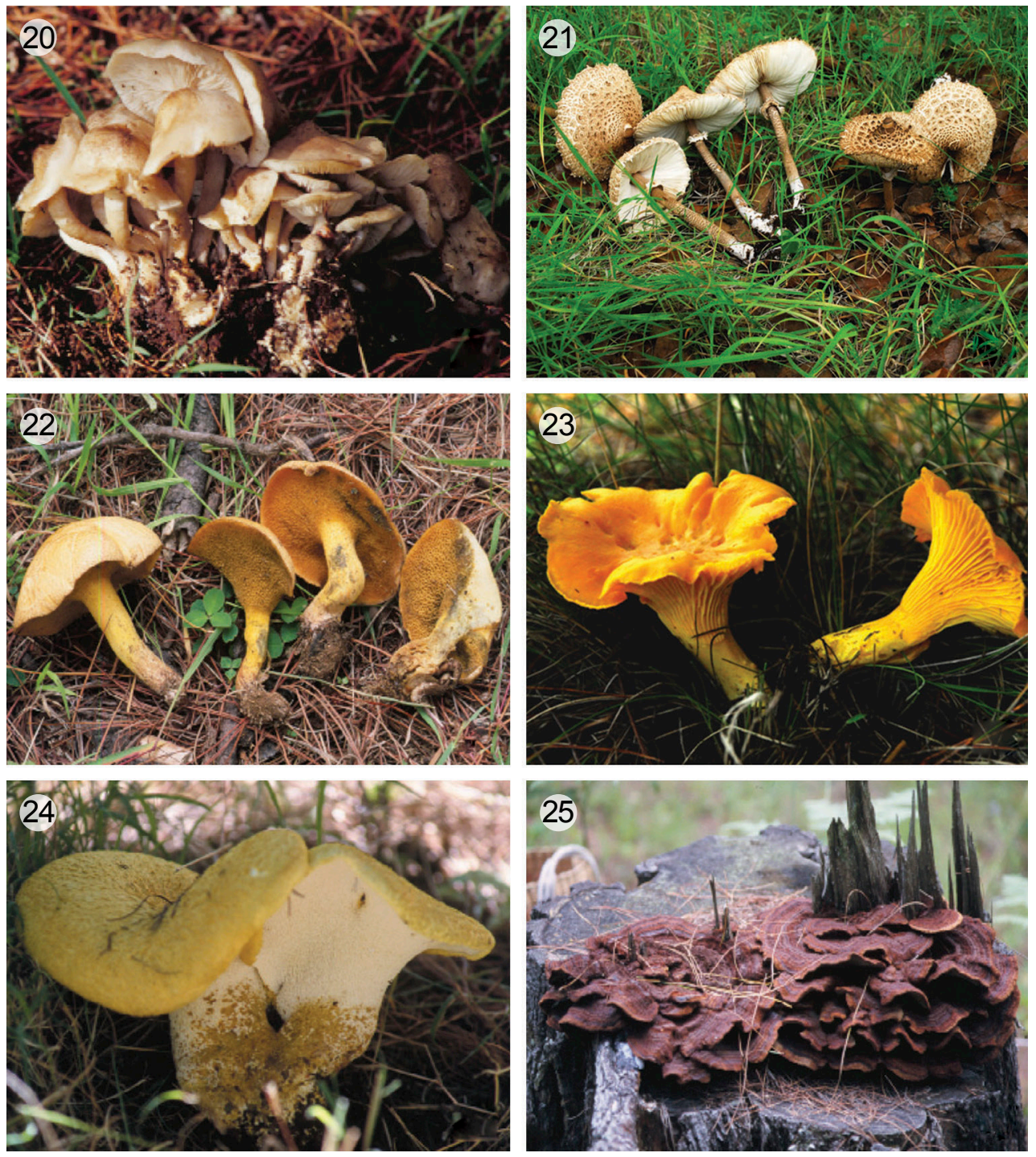

Figs. 20-25. 20. Lyophyllum decastes. 21. Macrolepiota procera. 22. Suillus tomentosus. 23. Cantharellus cibarius. 24. Albatrellus ellisii. 25. Phaeolus schweinitzii. 


\section{AGRADECIMIENTOS}

Se reconoce el apoyo de la Universidad de Guadalajara, a través de los proyectos P3e 34961 y 62935. Las recolectas se llevaron a cabo gracias al patrocinio económico de Ismael Gándara Mota, por lo que la primera autora reconoce su colaboración. Se agradece también la valiosa ayuda de Marko Aurelio Gómez-Hernández en las salidas al campo, en el trabajo de gabinete, en la revisión preliminar del escrito, y por las fotografías de las Figs. 18, 19 y 20. Se dan las gracias a Luis Villaseñor por el apoyo en algunas de las exploraciones y por las fotografías de las Figs. 22, 24 y 25, y a Adrián Galván Corona por proporcionar las fotografías de las Figs. 16, 17, 21 y 23. A Ricardo Valenzuela se le reconoce la asesoría en la determinación de gran parte de los poliporáceos. María de Jesús Herrera-Fonseca en IBUG, y Florencia Ramírez-Guillén y Juan Lara Carmona en XAL colaboraron en el trabajo de gabinete.

\section{LITERATURA CITADA}

Álvarez, I. y L. Guzmán-Dávalos. 1988. Nuevos registros de líquenes de Jalisco. Rev. Mex. Mic. 4: 89-96.

Álvarez, I. y L. Guzmán-Dávalos. 1993. Additions to the lichen flora from the State of Jalisco (Mexico). Mycotaxon 48: 359-370.

Álvarez, I. y L. Guzmán-Dávalos. 2009. Flavopunctelia y Punctelia (Ascomycetes liquenizados) de Nueva Galicia, México. Rev. Mex. Mic. 29: 15-29.

Bas, C. 1969. Morphology and subdivision of Amanita and monograph of its section Lepidella. Persoonia 5: 285-588.

Bigelow, H. E. 1982. North American species of Clitocybe, part II. J. Cramer. Berlin, Alemania. $281 \mathrm{pp}$.

Breitenbach, J. y F. Kränzlin. 1986. Fungi of Switzerland, Vol. 2. Verlag Mykologia. Lucerna, Suiza. 412 pp.

Breitenbach, J. y F. Kränzlin. 1991. Fungi of Switzerland, Vol. 3. Verlag Mykologia. Lucerna, Suiza. $361 \mathrm{pp}$.

Carrión, G. y M. Galván. 1984. Hongos fitopatógenos del estado de Veracruz. Bol. Soc. Mex. Mic. 19: 15-64.

Chacón, S. y G. Guzmán. 1983. Ascomycetes poco conocidos en México. Bol. Soc. Mex. Mic. 18: 183-218.

Dennis, R. W. G. 1981. British Ascomycetes. J. Cramer. Vaduz, Alemania. 585 pp.

Ferrusquía-Villafranca, I. 1993. Geology of Mexico: a synopsis. In: Ramamoorthy, T. P., R. Bye, A. Lot, J. Fa (eds.). Biological diversity of Mexico: origins and distribution. Oxford University Press. New York, USA. pp. 3-107. 
Fierros, M. L. y L. Guzmán-Dávalos. 1995. Inventario preliminar de los hongos macroscópicos de la Sierra de Quila, Jalisco, México. Bol. IBUG 3(1-3): 129-142.

Gilbertson, R. L. y L. Ryvarden. 1986. North American polypores, part I. AbortiporusLindtheria. Fungiflora. Oslo, Noruega. 433 pp.

Gilbertson, R. L. y L. Ryvarden. 1987. North American polypores, part II. MegasporoporiaWrightoporia. Fungiflora. Oslo, Noruega. 452 pp.

Gómez, M. A. 2004. Etnomicología en el poblado de Tapalpa, municipio de Tapalpa, Jalisco, México. Tesis profesional. Universidad de Guadalajara. Zapopan, México. 70 pp.

Guzmán, G. 1970. Monografía del género Scleroderma. Pers. emend. Fr. Darwiniana 16: 233-407.

Guzmán, G. 1977. Identificación de los hongos comestibles, venenosos, alucinantes y destructores de la madera. Limusa. México, D.F., México. 452 pp.

Guzmán, G. 1983. The genus Psilocybe (Beih. Nova Hedwigia 74). J. Cramer. Vaduz, Alemania. 439 pp.

Guzmán, G. 1994. Los hongos en la medicina tradicional de Mesoamérica y de México. Rev. Iberoamer. Mic. 11: 81-85.

Guzmán, G. 1998. Las especies del género Psilocybe (Fungi, Basidiomycotina, Agaricales) conocidas de Jalisco (México) y descripción de dos nuevas para la ciencia. Acta Bot. Mex. 43: 23-32.

Guzmán, G. 2008. Diversity and use of traditional Mexican medicinal fungi. A review. Inter. Journ. Med. Mushrooms 10: 209-217.

Guzmán, G. y D. A. García-Saucedo. 1973. Macromicetos del estado de Jalisco. I: Consideraciones generales y distribución de las especies conocidas. Bol. Soc. Mex. Mic. 7: 129-143.

Guzmán, G. y A. M. Pérez-Patraca. 1972. Las especies conocidas del género Panaeolus en México. Bol. Soc. Mex. Mic. 6: 17-53.

Guzmán, G., L. Montoya-Bello y V. M. Bandala. 1988. Nuevos registros de hongos alucinógenos del género Psilocybe en México y análisis de la distribución de las especies conocidas. Rev. Mex. Mic. 4: 255-265.

Guzmán, G. y F. Ramírez-Guillén. 2001. The Amanita caesarea-complex. J. Cramer. Stuttgart, Alemania. 66 pp.

Guzmán-Dávalos, L. e I. Álvarez. 1987. Observaciones sobre los líquenes de Jalisco y de Chiapas. Rev. Mex. Mic. 3: 217-230.

Guzmán-Dávalos, L. y G. Guzmán. 1979. Estudio ecológico comparativo entre los hongos (macromicetos) de los bosques tropicales y los de coníferas del sureste de México. Bol. Soc. Mex. Mic. 13: 89-125.

Guzmán-Dávalos, L. y G. Guzmán. 1985. Hongos del estado de Jalisco, V. El género Scleroderma. Rev. Mex. Mic. 1: 109-128.

Guzmán-Dávalos, L. y G. Guzmán. 1986. Hongos del estado de Jalisco, VII. El género Gymnopilus (Cortinariaceae). Rev. Mex. Mic. 2: 157-185.

Guzmán-Dávalos, L. y G. Guzmán. 1995. Toward a monograph of the genus Gymnopilus (Cortinariaceae) in Mexico. Doc. Mycol. 25(98-100): 197-212.

Guzmán-Dávalos, L. y F. Trujillo. 1984. Hongos de Jalisco, IV. Nuevos registros. Bol. Soc. Mex. Mic. 19: 319-326. 
Guzmán-Dávalos, L., O. Rodríguez, M. R. Sánchez-Jácome y S. Chacón. 2001 (2003). Ascomycotina conocidos de Jalisco, México. Bol. IBUG 9(1-2): 11-23.

Herrera-Fonseca, M., L. Guzmán-Dávalos y O. Rodríguez. 2002. Contribución al conocimiento de la micobiota de la región de San Sebastián del Oeste, Jalisco, México. Acta Bot. Mex. 58: 19-50.

López, A., D. Martínez y J. García. 1980. Phallales conocidos del estado de Veracruz. Bol. Soc. Mex. Mic. 14: 39-49.

Mains, E. B. 1958. North American entomogenous species of Cordyceps. Mycologia 50: 169-222.

Manzi, J. 1976. Hongos. Contribución al conocimiento de las especies comestibles y venenosas del área central de Jalisco, México. Ed. Combonianas. Guadalajara, México. 119 pp.

Martin, G. W. y C. J. Alexopoulos. 1969. The Myxomycetes. University of Iowa Press. Iowa City, EUA. 480 pp. + 41 láms.

Montoya, L., V. M. Bandala y G. Guzmán. 1996. New and interesting species of Lactarius from Mexico including scanning electron microscope observations. Mycotaxon 57: 411-424.

Mora, V. 1985. Nuevos registros del género Volvariella en Morelos. Rev. Mex. Mic. 1: 91100.

Moser, M. 1983. Keys to agarics and boleti. Phillips. Londres, Inglaterra. 535 pp.

Mueller, G. M. 1992. Systematics of Laccaria (Agaricales) in the continental United States and Canada, with discussions on extralimital taxa and descriptions of extant types. Fieldiana Bot. 30: 1-158.

Pegler, D. N. 1977. A preliminary agaric flora of East Africa. Kew Bull. 6: 1-615.

Rodríguez, O. 1998. Avances en el conocimiento del género Pluteus (Fungi, Agaricales) en Nueva Galicia, México. Bol. IBUG 5(1-3): 87-93.

Rodríguez, O. y L. Guzmán-Dávalos. 2000. Algunas especies del género Pluteus Fr. (Pluteaceae, Agaricales) citados de Nueva Galicia, México. Bol. IBUG 7(1-3): 61-77.

Rodríguez, O. y L. Guzmán-Dávalos. 2001. Clave dicotómica de las especies del género Pluteus Fr. (Pluteaceae) conocidas de la región de la Nueva Galicia, y algunas áreas aledañas de México. Acta Bot. Mex. 57: 23-36.

Rodríguez, O., M. Garza y L. Guzmán-Dávalos. 1994. Inventario preliminar de los hongos del Volcán de Tequila, estado de Jalisco, México. Rev. Mex. Mic. 10: 103-111.

Rodríguez, O., L. Villaseñor, M. Cedano y A. Arias. 2002. Hongos del Bosque La Primavera. Guía ilustrada. Departamento de Botánica y Zoología, Centro Universitario de Ciencias Biológicas y Agropecuarias, Universidad de Guadalajara. Zapopan, México. 109 pp.

Rzedowski, J. 1978. La vegetación de México. Limusa. México, D.F., México. 432 pp.

Sánchez-Jácome, M. R. y L. Guzmán-Dávalos. 1997. Nuevos registros de Thelephora (Aphyllophorales, Basidiomycotina) para México. Rev. Mex. Mic. 13: 70-77.

Smith, A. H. 1951. The North American species of Naematoloma. Mycologia 43: 467-521.

Téllez, C., L. Guzmán-Dávalos y G. Guzmán. 1988. Contribución al conocimiento de los hongos de la Reserva de la Biosfera de la Sierra de Manantlán, Jalisco. Rev. Mex. Mic. 4: 123-130. 
Trujillo, F., M. Castañeda y L. Guzmán-Dávalos. 1986. Hongos del estado de Jalisco, VI. Los Myxomycetes conocidos. Tiempos de Ciencia 5: 42-51.

Vargas, O., L. Guzmán-Dávalos y L. S. Vázquez. 1992. Nuevos registros de Basidiomycetes para Jalisco. Bol. IBUG 1(4): 307-319.

Vargas, O., L. Guzmán-Dávalos y L. S. Vázquez. 1993. Observations on some little known macrofungi from Jalisco (Mexico). Mycotaxon 49: 437-447.

Vázquez, L. S. y L. Guzmán-Dávalos. 1988. Algunas especies de hongos de la Barranca de Huentitán, estado de Jalisco. Rev. Mex. Mic. 4: 75-88.

Vázquez, L. S. y L. Guzmán-Dávalos. 1991a. Nuevos registros de poliporáceos estipitados de Jalisco. Rev. Mex. Mic. 7: 113-120.

Vázquez, L. S. y L. Guzmán-Dávalos. 1991b. Los hongos del género Volvariella (Agaricales, Basidiomycetes) conocidos en Jalisco. Bol. IBUG 1(1): 15-22.

Vázquez, L. S., L. Guzmán-Dávalos y G. Guzmán. 1989. Contribución al conocimiento de las especies del género Volvariella en Jalisco. Rev. Mex. Mic. 5: 169-179.

Recibido en julio de 2010.

Aceptado en febrero de 2012. 


\section{APÉNDICE}

Especies estudiadas de la región de Tapalpa. $\mathrm{A}=$ alucinógeno, $\mathrm{BE}=$ bosque de encino, $\mathrm{BP}=$ bosque de pino, $\mathrm{BPE}=$ bosque de pino-encino, $\mathrm{C}=$ comestible, $\mathrm{DM}$ = destructor de la madera, $\mathrm{F}$ = fimícola, $\mathrm{Fi}$ = fitopatógeno, $\mathrm{Fu}=$ fungícola, $\mathrm{H}=$ humícola, $\mathrm{I}=$ entomopatógeno, $\mathrm{L}=$ lignícola, $\mathrm{M}=$ micorrizógeno, $\mathrm{Me}=$ medicinal, $\mathrm{PA}$ $=$ pastizal, $\mathrm{T}=$ terrícola, $\mathrm{V}=$ venenoso, $\mathrm{W}=$ micromicete. Los nombres al final de cada especie, después de las referencias, hacen alusión a los colectores y sus números. * significa nuevo registro para Jalisco.

Myxomycota
Hemitrichia calyculata (Speg.) M.L. Farr;
BP, L (Castañeda 65)
Leocarpus fragilis (Dicks.) Rostaf.; BP, L
(Castañeda 62)

Lepidoderma tigrinum (Schrad.) Rostaf.; BP, L (Castañeda 63)

Lycogala epidendrum (J.C. Buxb. ex L.) Fr.; BP, C, L (Gándara 568, Gómez 109, Luna 12)

L. flavofuscum (Ehrenb.) Rostaf.; BP, BPE, L (Gándara 306, Guzmán-Dávalos 3869)

Tubifera ferruginosa (Batsch) J.F. Gmel.; BPE, L (Sánchez-Jácome 657)

Ascomycota

Elaphomycetales

Elaphomyces granulatus Fr.; BPE, C, Me, T (Guzmán-Dávalos 5876)

Hypocreales

Apiocrea hyalina (Schwein.) Syd. \& P. Syd., anamorfo de Hypomyces hyalinus (Schwein.) Tul. \& C. Tul.; BPE, Fu, W (Guzmán-Dávalos 3111)

Cordyceps capitata (Holmsk.) Link; A, BPE, Fu (Guzmán-Dávalos 5875)

C. militaris (L.) Fr.; BPE, I (GuzmánDávalos 5882)

Hypomyces lactifluorum (Schwein.) Tul. \& C. Tul.; BP, BPE, C, Fu, Me, W (Gándara 203, 303, 342, 688, Gómez 91) (Fig. 16) Sepedonium chrysospermum (Bull.) Fr., anamorfo de Hypomyces chrysospermum Tul. \& C. Tul.; BP, Fu, W (Gándara 337)
Leotiales

Chlorociboria aeruginosa (Oeder) Seaver ex C.S. Ramamurthi, Korf \& L.R. Batra; BPE, DM, L (Lugo 1)

Pezizales

Aleuria aurantia (Pers.) Fuckel; BPE, C, T (Rodríguez 698)

Helvella acetabulum (L.) Quél.; BPE, C, M, T (Guzmán-Dávalos 2244, Loeza 194)

H. atra J. König; BPE, C, M, T (Guzmán-

Dávalos 2189)

H. crispa (Scop.) Fr.; BP, BPE, C, M, T (Gándara 142, Guzmán-Dávalos 2187, 5486, López 18, Nieves 37) (Fig. 17)

H. elastica Bull.; BP, BPE, C, M, T (Acosta

6, Gándara 685)

H. macropus (Pers.) P. Karst.; BP, BPE, C, M, T (Alquiciras 22, 111, Gándara 127, 336, 647)

H. pezizoides Afzel.; BPE, C, M, T

(González s.n., Guzmán-Dávalos 2224)

H. subglabra N.S. Weber; BPE, C, M, T

(Álvarez 720, Arreaga 31)

Humaria hemisphaerica (F.H. Wigg.)

Fuckel; BPE, M, T (Zamarripa 90)

Scutellinia scutellata (L.) Lambotte; BPE,

H, T (Rodríguez 1230)

Xylariales

Daldinia concentrica (Bolton) Ces. \& De

Not.; BP, DM, L, Me (Gómez 87)

D. vernicosa Ces. \& De Not.; BP, DM, L

(Gándara 165-A, Vargas 38) 
Apéndice. Continuación.

Discoxylaria myrmecophila J.C. Lindq. \& J.E. Wright; BPE, H (sobre hormigueros de Atta) (Gándara 22)

Hypoxylon thouarsianum (Lév.) Lloyd; BP, BPE, DM, L (De Luna 40, Godoy s.n., Guzmán-Dávalos 1456, Lugo 17, Zúñiga 23)

Xylaria hypoxylon (L.) Grev.; BPE, DM, L (Fallad 27, Guzmán-Dávalos 3871)

Basidiomycota

Agaricales

Hygrophoraceae

*Hygrophorus chrysodon (Batsch) Fr.; BP,

C, M, T (Guzmán-Dávalos 8972)

Tricholomataceae

Armillaria polymyces (Pers.) Singer \&

Clémençon; BP, C, Fi, DM (Guzmán-

Dávalos 3117, Nieves 150)

Baeospora myosura (Fr.) Singer; BP, L

(Sánchez-Jácome 652)

Clitocybe gibba (Pers.) P. Kumm.; BP, BPE,

C, M, Me, T (Castro 31, Gándara 96, 146, 189, Gómez 75)

*C. hydrogramma (Bull.) P. Kumm.; BP, H

(Gándara 168, Gómez 86-B) (Figs. 9-10)

Cyptotrama asprata (Berk.) Redhead \&

Ginns; BPE, L (Guzmán-Dávalos 3876)

Dictyopanus pusillus var. rhiphidium

(Berk.) Singer; BP, DM, L (Guzmán-

Dávalos 2219, 2257)

Gymnopus polyphyllus (Peck) Halling; BE,

BP, H (Alquiciras 282, Gándara 291)

Hohenbuehelia angustata (Berk.) Singer;

BPE, C, L (Guzmán-Dávalos 3852, 8767)

H. atrocaerulea (Fr.) Singer; BPE, C, DM,

L (Romero 18-A)

Laccaria amethystina Cooke; BP, BPE, C, M, T (Gándara 108, 138, 179, 198, 352)

L. laccata (Scop.) Cooke; BP, BPE, C, M, T (Acosta 13, Castro 1, Guzmán-Dávalos 5364, 5487)
*L. ochropurpurea (Berk.) Peck; BP, BPE, C, M, T (Gándara 119, Gómez 98, 107) (Figs. 12-13)

Lepista nuda (Bull.) Cooke; BP, BPE, C, T (Gándara 128, Gómez 9, Guzmán-Dávalos 3127)

Lyophyllum decastes (Fr.) Singer; BP, C, T (Gándara 322, González-Villarreal 2610)

(Fig. 20)

Marasmius oreades (Bolton) Fr.; BP, BPE, C, H, PA (Álvarez 731, García-Saucedo 219, Meza 12, Palacios 11)

M. rotula (Scop.) Fr.; BP, BPE, L (Bauche 3, Buenrostro 25, Dávalos 1, Gándara 85 , Loeza 212)

*Megacollybia platyphylla (Pers.) Kotl. \&

Pouzar; BP, H (Gándara 701, 717)

Mycena leaiana (Berk.) Sacc.; BPE, L

(Motolinia 25)

M. pura (Pers.) P. Kumm.; BP, BPE, C, H

(Álvarez 725, Gándara 680, 700)

Xeromphalina campanella (Batsch) Maire; BP, L (Gándara 143, 316, 348, Gómez 88, 110)

$X$. tenuipes (Schwein.) A.H. Sm.; BPE, L

(García-Niño 10, Guzmán-Dávalos 3849)

Amanitaceae

*Amanita austrostraminea D.A. Reid; BP, M, T (Gándara 185) (Figs. 2-7)

A. chlorinosma (Peck) Lloyd; BPE, M, T, V

(Gándara 112)

A. citrina (Schaeff.) Pers.; BP, M, T, V

(Gándara 575, 580, 587)

A. cokeri (E.-J. Gilbert \& Kühner) E.-J.

Gilbert; BP, M, T, V (Gándara 160, 358)

A. fulva Fr.; BP, BPE, C, M, T (Gándara

294, Gómez 67, 70, Lugo 6, Soltero s.n.)

A. gemmata (Fr.) Bertill.; BP, BPE, M, T, V

(Gándara 293, 329, 346, 350, Gómez 44)

A. laurae Guzmán \& Ram.-Guill.; BP, C, M, Me, T (Castillo-Suárez 27, Gómez 42, 90) (Fig. 18) 
Apéndice. Continuación.

A. magnivelaris Peck; BPE, M, T, V

(Alquiciras 120)

A. muscaria var. flavivolvata (Singer)

D.T. Jenkins; A, BP, BPE, M, Me, T, V (Gándara 7, 308, 562, 576, Gómez 100)

A. onusta (Howe) Sacc.; BP, BPE, M, T, V

(Arreaga-Pérez 18, Gándara 57, 105)

A. pantherina (DC.) Krombh. var. pantherina; BP, M, T, V (Gómez 65)

*A. pantherina var. velatipis (G.F. Atk.)

D.T. Jenkins; BP, M, T, V (Gándara 398)

A. roseotincta (Murrill) Murrill; BP, BPE,

M, T (Gándara 159, Guzmán-Dávalos 5370)

A. rubescens Pers.; BE, BP, BPE, C, M,

T (Gándara 175, 290, Rodríguez 810-B, Téllez 963)

A. tecomate Guzmán \& Ram.-Guill.; BP, C, M, Me, T (Gándara 80, Gómez 91, 96, Ramírez 1397) (Fig. 19)

*A. tullossii Guzmán \& Ram.-Guill.; BP, C, M, Me, T (Gándara 309) (Fig. 8)

A. vaginata (Bull.) Lam.; BP, BPE, C, M,

T (Gándara 163, 287, Guzmán-Dávalos 3136, 5886)

A. valens (E.-J. Gilbert) Bertault; BPE, M, T (Loeza 202)

A. virosa (Fr.) Bertill.; BPE, M, T, V

(Guzmán-Dávalos 5470)

Pluteaceae

Pluteus cervinus (Schaeff.) P. Kumm.; BP,

C, L (Rodríguez 2459)

P. petasatus (Fr.) Gillet; BP, L, C

(Rodríguez 2458)

Volvariella bombycina (Schaeff.) Singer;

BP, C, L (Ortiz 10)

*V. cinerea (Beardslee) Mora ex Guzmán;

BP, L (Gándara 186) (Fig. 14)

Agaricaceae

*Agaricus augustus Fr.; BPE, C, T (Dávalos 24)
A. campestris L.; BP, C, PA, T (Dávalos s.n., Gándara 297, Gómez 6, Plascencia 2, Villaseñor 307)

A. xanthodermus Genev. var. xanthodermus; BPE, T, V (Gándara 180, Herrera 963)

Chlorophyllum molybdites (G. Mey.)

Massee; BPE, PA, T, V (Gándara 34, Pérez 9, Zermeño s.n.)

Cystoderma fallax A.H. Sm. \& Singer; BP,

L (Gándara 137)

Leucoagaricus rubrotinctus (Peck) Singer; BP, BPE, T (Álvarez 721, Gándara 65, García-Niño 7, González-Paredes 16)

Macrolepiota procera (Scop.) Singer; BP, C, T (Gándara 33, 34-B, 314, 331, 359)

(Fig. 21)

Coprinaceae

Coprinus comatus (O.F. Müll.) Pers.; BP, BPE, C, H, PA (Gándara 35, 298, Gómez 43, Meza 13)

Panaeolina foenisecii (Pers.) Maire; F, PA, V (Guzmán-Dávalos 6403)

Panaeolus antillarum (Fr.) Dennis; F, PA, V (Gándara 69, 299, Guzmán-Dávalos 5704) P. sphinctrinus (Fr.) Quél.; BP, BPE, F, PA, V (Gándara 28-A, 319, Guzmán-Dávalos 3126, 5355, 5870)

P. subbalteatus (Berk. \& Broome) Sacc.; F, PA, V (Álvarez 18)

Psathyrella candolleana (Fr.) Maire; BPE, H (Guzmán-Dávalos 2186, Rodríguez 690)

P. velutina (Pers.) Singer; BPE, H (Bauche 6) Bolbitiaceae

*Conocybe tenera (Schaeff.) Fayod; BPE, PA, T, V (Alquiciras 5, Blanco 54)

Strophariaceae

Hypholoma fasciculare (Huds.) P. Kumm.; BP, BPE, H, V (Castro 11, Gándara 318, Guzmán-Dávalos 5685-A, 5692, Hernández-Hernández 28) 
Apéndice. Continuación.

*H. subviride (Berk. \& M.A. Curtis)

Dennis; BP, H, V (Gándara 131) (Fig. 11)

Psilocybe coprophila (Bull.) P. Kumm.;

BP, BPE, F, V (Arreaga 4, Gándara 193, González s.n., Guzmán-Dávalos 5375,

Nieves s.n.)

P. cubensis (Earle) Singer; A, BPE, F, PA

(Guzmán 21997, Reyes 3)

P. mexicana R. Heim; A, BP, PA, T (Guzmán

21999, Martínez s.n., Pérez de la Rosa s.n.)

Stropharia coronilla (Bull. ex DC.) Quél.;

BP, BPE, C, PA, T (Álvarez 32, Gándara

25, 173, Gómez 30, Pérez-Fabián 11)

S. semiglobata (Batsch) Quél.; BE, BP,

BPE, F, PA, V (Gándara 172, 300, 335,

Gómez 50-A, 54)

Cortinariaceae

Inocybe fastigiata (Schaeff.) Quél.; BPE,

M, T, V (Álvarez 1137)

Crepidotaceae

Crepidotus mollis (Schaeff.) Staude; BPE,

DM, L (Guzmán-Dávalos 2250, 3123)

C. uber (Berk. \& M.A. Curtis) Sacc.; BPE,

DM, L (Alquiciras 116-B)

Paxillaceae

Hygrophoropsis aurantiaca (Wulfen)

Maire; BP, C, M, T (Gándara 29-A, 307, 341, 364, 672)

Omphalotus mexicanus Guzmán \& V. Mora;

BE, T, V (Sánchez-Jácome 252)

Paxillus panuoides (Fr.) Fr.; BPE, DM, L

(Loeza 215)

*Pseudomerulius aureus (Fr.) Jülich; BPE,

DM, L (Romero 14-B)

Gomphidiaceae

Gomphidius rutilus (Schaeff.) S. Lundell;

BPE, C, M, T (Castillo-Suárez s.n.,

Vázquez-Morales 22)

Boletaceae

Boletellus coccineus (Sacc.) Singer; BPE,

C, M, T (Sánchez-Jácome 161)
*Boletus ferrugineus Schaeff.; BPE, C, M, T (Téllez 997)

B. flammans E.A. Dick \& Snell; BP, M, T, V (Gándara 76)

B. frostii J.L. Russell; BP, C, M, T

(Rodríguez 808)

Meiorganum curtisii (Berk.) Singer, J.

García \& L.D. Gómez; BP, DM, L (Gómez

79, Guzmán-Dávalos 5689-A, Rivera s.n.)

Phylloporus rhodoxanthus (Schwein.) Bres.;

BE, C, M, T (Rodríguez 815)

Strobilomyces confusus Singer; BP, C, M, T

(Gándara 134)

S. strobilaceus (Scop.) Berk.; BP, C, M, T

(Gándara 334)

*Suillus acidus (Peck) Singer; BP, C, M, T

(Gándara 26)

S. americanus (Peck) Snell; BP, C, M, T

(Gándara 399)

S. brevipes (Peck) Kuntze; BP, C, M, Me, T

(Gándara 323, Gómez 5)

S. cothurnatus var. hiemalis (Singer) A.H.

Sm. \& Thiers; BP, BPE, C, M, T (Gómez 97, Guzmán-Dávalos 5758)

S. granulatus (L.) Roussel; BP, C, M, Me, T (Gándara 288, 330, Gómez 14)

S. luteus (L.) Roussel; BPE, C, M, Me, T (Loeza 190)

S. tomentosus (Kauffman) Singer; BP, C,

M, T (Gándara 188, 321, 332, 339, Gómez 64) (Fig. 22)

Bondarzewiaceae

Bondarzewia berkeleyi (Fr.) Bondartsev \&

Singer; BPE, C, L (Silva \& Arreaga s.n.)

Russulaceae

Lactarius deliciosus (L.) Gray var. deliciosus; BP, C, M, Me, T (Gándara 132, Guzmán-Dávalos 8757, 8779, Herrera 961, 973)

L. indigo (Schwein.) Fr.; BP, C, M, Me, T (Gándara 100) 
Apéndice. Continuación.

L. piperatus (L.) Pers.; BPE, C, M, T

(Guzmán-Dávalos 5872)

L. scrobiculatus (Scop.) Fr.; BPE, C, M, T

(Téllez 249)

Russula alutacea (Fr.) Fr.; BPE, C, M, T

(Dávalos s.n.)

R. brevipes Peck var. brevipes; BPE, C, M,

$\mathrm{T}$ (Ramírez-Delgadillo 1399)

R. cyanoxantha (Schaeff.) Fr.; BPE, C, M, T

(Téllez 241)

R. foetens Pers.; BPE, M, T, V (Guzmán-

Dávalos 2200)

$R$. nigricans Fr.; BPE, C, M, T (Téllez 998)

R. olivacea (Schaeff.) Fr.; BPE, C, M, T

(Pérez de la Rosa s.n.)

Auriculariales

Auricularia fuscosuccinea (Mont.) Henn.;

BPE, C, DM, L, Me (Munguía-Matute 12)

A. polytricha (Mont.) Sacc.; BPE, C, DM, L

(Guzmán-Dávalos 3881, Ruiz 15, Uribe 17)

Cantharellales

Cantharellaceae

Cantharellus cibarius Fr. var. cibarius; BP, BPE, C, M, T (Alcalá-Arce 2, Alquiciras

110, Gándara 355, Medina 12, Villaseñor

511) (Fig. 23)

C. cinnabarinus (Schwein.) Schwein.; BPE,

C, M, T (Guzmán-Dávalos 5890)

Clavariadelphaceae

Clavariadelphus pistillaris (L.) Donk; BPE, C, T (Nieves s.n.)

Scutigeraceae

Albatrellus cristatus (Schaeff.) Kotl. \&

Pouzar; BE, T (Rodríguez 809)

A. ellisii (Berk.) Pouzar; BP, T (Villaseñor

510) (Fig. 24)

*Polyporoletus sublividus Snell; BPE, M, T

(Guzmán-Dávalos 2201)

Sparassidaceae

Sparassis crispa (Wulfen) Fr.; BPE, C, L

(Guzmán-Dávalos 3849, Sánchez-Jácome 163)
Dacrymycetales

Dacrymyces dictyosporus G.W. Martin; BP,

DM, L (Gándara 67, 125)

Dacryopinax spathularia (Schwein.) G.W.

Martin; BP, C, DM, L (Gándara 86, 106,

118, Guzmán-Dávalos 8987)

Fistulinales

Fistulina guzmanii Brusis; BP, C, DM, L

(Bayardo s.n.)

Ganodermatales

Ganoderma applanatum (Pers.) Pat.; BPE,

DM, L (Dávalos s.n., Guzmán-Dávalos

2212, 2260, Loeza 195, 208)

G. curtisii (Berk.) Murrill; BPE, DM, L

(Gándara 136, 162, Gómez 38, 48, Oliva 29)

G. lobatum (Schwein.) G.F. Atk.; BE, BP,

DM, L (Castro 12, Guzmán-Dávalos 2231, 3864, Vargas 449, Villarreal de Puga 913)

G. grupo lucidum (Curtis) P. Karst.; BPE,

DM, L, Me (Manzano-Ochoa s.n.)

G. resinaceum Boud.; BPE, DM, L

(Guzmán-Dávalos 2211, Romero 15)

Hericiales

Auriscalpium vulgare Gray; BP, BPE, L

(Cruz-Gómez 22, Gándara 11, 304, 719,

Gómez 4)

Hymenochaetales

Coltricia cinnamomea (Jacq.) Murrill; BP, T

(Gándara 1)

C. focicola (Berk. \& M.A. Curtis) Murrill;

BP, BPE, T (Gándara 2, 89, 123, Guzmán-

Dávalos 2227, Zermeño s.n.)

C. perennis (L.) Murrill; BP, BPE, T

(Gándara 92, Godoy s.n., Gómez 55, 62,

Rodríguez 1795-A)

Coltriciella dependens (Berk. \& M.A.

Curtis) Murrill; BP, L (Castañeda 70)

Hymenochaete sallei Berk. \& M.A. Curtis;

BP, DM, L (Guzmán-Dávalos 1458)

Inonotus fulvomelleus Murrill; BP, DM, L

(Peña 21) 
Apéndice. Continuación.

Phellinus gilvus (Schwein.) Pat.; BPE, DM, L (Dávalos s.n., Dávila 10, Ruiz 7, SuárezPlascencia s.n., Tinajero 2)

P. sarcites (Fr.) Ryvarden; BPE, DM, L (Álvarez 72)

Lycoperdales

Geastraceae

Geastrum pectinatum Pers.; BPE, H

(Villela-Gaytán 5)

*G. quadrifidum DC. ex Pers.; BP, H (Sánchez 12)

G. saccatum Fr.; BP, H, Me (Rodríguez 703)

G. velutinum Morgan; BP, BPE, $\mathrm{H}$ (Alquiciras 132, Castro 10, López 7)

Lycoperdaceae

Calvatia cyathiformis (Bosc) Morgan; BPE, C, Me, PA, T (Blanco 57, Gómez

16, Guzmán-Dávalos 5702, Montiel 12,

Toscano 71)

Lycoperdon marginatum Vittad.; BP, BPE, C, H, Me, PA (Gándara 17, 37, 139, 704, Guzmán-Dávalos 3157)

L. perlatum Pers.; BP, C, H, Me (Gándara 27, 724, Gómez 95)

L. pyriforme Schaeff.; BP, C, L, Me (Nieves 155)

Phallales

*Phallus ravenelii Berk. \& M.A. Curtis; BP, $\mathrm{T}$ (Gándara 3) (Fig. 15)

Poriales

Coriolaceae

Abortiporus biennis (Bull.) Singer; BPE,

DM, L (Zamarripa 84)

Bjerkandera adusta (Willd.) P. Karst.; BP, BPE, DM, L (Gándara 152, 349, GuzmánDávalos 2252, Loeza 210, Rodríguez 684)

Climacocystis borealis (Fr.) Kotl. \& Pouzar; BPE, DM, L (Vargas 446)

Coriolopsis gallica (Fr.) Ryvarden; BPE, DM, L (Fanti 484)
C. rigida (Berk. \& Mont.) Murrill; BP, BPE, DM, L (Gómez-Martínez s.n., GonzálezParedes 19)

Cryptoporus volvatus (Peck) Shear; BPE, DM, L (Dávalos 51, Guzmán-Dávalos 3758, Iñiguez s.n.)

Funalia polyzona (Pers.) Niemelä; BPE, DM, L (Oliva 28)

*Gloeophyllum mexicanum (Mont.) Ryvarden; BP, BPE, DM, L (Gándara 21, 91, 121, 133, Guzmán-Dávalos 2235)

G. sepiarium (Wulfen) P. Karst.; BP, DM, L (Alquiciras 112, Gándara 312, 343, 360, 720)

G. striatum (Fr.) Murrill; BP, DM, L

(Alquiciras 113)

Heterobasidion annosum (Fr.) Bref.; BPE, DM, L (López 9)

Hexagonia cucullata (Mont.) Murrill; BPE, DM, L (Rivera-Sánchez 6)

H. hirta (P. Beauv.) Fr.; BPE, DM, L

(García-Saucedo 418, Ramírez-Hernández

s.n., Rodríguez 14)

H. tenuis (Hook.) Fr.; BP, DM, L (Gándara 164, Gómez 101, González-Paredes 15, Oliva 30)

Hydnopolyporus fimbriatus (Cooke) D.A.

Reid; BP, BPE, L (Acosta 7, Arreaga-Pérez 29, Gándara 8)

Lenzites betulina (L.) Fr.; BP, BPE, DM, L

(Gándara 4, Loeza 201)

*Pachykytospora papyracea (Cooke)

Ryvarden; BP, DM, L (Cruz s.n.)

Phaeolus schweinitzii (Fr.) Pat.; BP, DM, L (Alquiciras 21, Álvarez 20, Arreaga 36, Gándara 338, 351) (Fig. 25)

Porodisculus pendulus (Fr.) Murrill; BPE, DM, L (Velazco 299)

Trametes cervina (Schwein.) Bres.; BP, BPE, DM, L (Guzmán-Dávalos 1467, Téllez 250-B) 
Apéndice. Continuación.

T. hirsuta (Wulfen) Lloyd; BP, BPE, DM, L (Álvarez 736, Guzmán-Dávalos 1463, Téllez 25-A)

T. maxima (Mont.) A. David \& Rajchenb.; BP, DM, L (Lomelí s.n., Sánchez 11) T. versicolor (L.) Lloyd; BP, BPE, DM, L, Me (Fortes 5, Guzmán-Dávalos 1460, 2251, 3158, Mac Gregor Fors 5)

T. villosa (Sw.) Kreisel; BP, BPE, DM, L (Alonso 11, 22, Ortiz 13)

Trichaptum abietinum (Dicks.) Ryvarden; BP, BPE, DM, L (Contreras 40, Gándara 9, 317, Guzmán-Dávalos 5763, Rodríguez 1040)

T. biforme (Fr.) Ryvarden; BP, BPE, DM, L (Guzmán-Dávalos 3171, Martínez 7, Sánchez 3, Valenzuela s.n., Villarreal de Puga s.n.)

T. sector (Ehrenb.) Kreisel; BP, DM, L (Gándara 737)

Tyromyces caesius (Schrad.) Murrill; BP, BPE, DM, L (Gándara 87, 345, Loeza 206, 214)

Lentinaceae

Lentinula boryana (Berk. \& Mont.) Pegler; BE, BP, BPE, C, DM, L (Alquiciras 3, Álvarez 871, Téllez 932)

Lentinus badius (Berk.) Berk.; BPE, DM, L (Guzmán-Dávalos 2232)

L. crinitus (L.) Fr.; BP, BPE, DM, L, Me (Alonso 19, Gándara 113, GuzmánDávalos 3865, Lozano 7, Pérez-Fabián 14) Neolentinus suffrutescens (Brot.) T.W. May \& A.E. Wood; BPE, C, DM, L (De Luna 24)

Pleurotus djamor (Rumph. ex Fr.) Boedijn; BPE, C, DM, L, Me (Vázquez 398)

P. levis (Berk. \& M.A. Curtis) Singer; BPE, C, DM, L (De Luna 25, Guzmán-Dávalos 3122)

Polyporaceae

Echinochaete brachypora (Mont.)
Ryvarden; BP, BPE, DM, L (De Luna 30, Gándara 310, González s.n., MaldonadoMoreno 1)

Favolus tenuiculus P. Beauv.; BPE, C, DM, L (Alonso 3, 18, Blanco-Cisneros 3, Nieves s.n.)

Panus conchatus (Bull.) Fr.; BPE, DM, L (Guzmán-Dávalos 3855)

Polyporus alveolaris (DC.) Bondartsev \& Singer; BP, DM, L (Gándara 583)

P. arcularius (Batsch) Fr.; BP, DM, L

(Gándara 6, 10, 36, 161, 174)

P. tricholoma Mont.; BP, BPE, DM, L (Alquiciras 123, Gándara 23, GuzmánDávalos 3860)

P. varius (Pers.) Fr.; BPE, DM, L (GuzmánDávalos 3861)

Schizophyllales

Schizophyllum commune Fr.; BP, BPE, C, DM, L, Me (Gándara 155, 311, GuzmánDávalos 8761, Orozco 24, Torres 401) Sclerodermatales Astraeus hygrometricus (Pers.) Morgan; BP, BPE, M, T (Castro 6, Gándara 114, Medina 2, Pérez de la Rosa s.n., PérezFabián 16)

Pisolithus arhizus (Scop.) Rauschert; BP, BPE, M, T (Gómez-Martínez s.n., Vázquez 400)

Scleroderma areolatum Ehrenb.; BPE, M, T, V (García-Niño 1, 8, Hernández-Conrique 17, Valdivia 18)

*S. citrinum Pers.; BP, M, T, V (GarcíaSaucedo s.n.)

S. polyrhizum (J.F. Gmel.) Pers.; BP, BPE, M, T, V (Bojovoe 16, Guzmán-Dávalos 5765)

S. texense Berk.; BPE, M, T, V (Guzmán-

Dávalos 2202)

S. verrucosum (Bull.) Pers.; BPE, M, T, V (Domínguez 24) 
Apéndice. Continuación.

\begin{tabular}{ll}
\hline Stereales & S. ochraceoflavum (Schwein.) Sacc.; BP, \\
Meruliaceae & DM, L (Gándara 178) \\
Byssomerulius incarnatus (Schwein.) Gilb.; & S. ostrea (Blume \& T. Nees) Fr.; BP, BPE, \\
BP, BPE, DM, L (Arreaga 45, Cárdenas & DM, L (Guzmán-Dávalos 5761, Sánchez \\
18, Gómez 84, Guzmán-Dávalos 5467) & 2, Téllez 251) \\
Gloeoporus dichrous (Fr.) Bres.; BP, DM, L & S. subpileatum Berk. \& M.A. Curtis; BPE, \\
(Nepote 14) & DM, L (Guzmán-Dávalos 5874) \\
G. thelephoroides (Hook.) G. Cunn.; BP, & Thelephorales \\
DM, L (Gándara 151, Orozco 6) & Thelephora caryophyllea (Schaeff.) Pers.; \\
Merulius tremellosus Schrad.; BPE, DM, & BPE, T (Guzmán-Dávalos 2225, 2226, \\
L (Pérez de la Rosa s.n., Rodríguez 697, & Mendoza 8) \\
699) & T. regularis Schwein. var. regularis; BPE, T \\
Peniophoraceae & (Guzmán-Dávalos 2253) \\
Peniophora albobadia (Schwein.) Boidin; & T. terrestris Ehrh.; BPE, M, T (Guzmán- \\
BP, BPE, DM, L (Langle 7, Lomelí s.n., & Dávalos 2247) \\
Rodríguez 700) & Tremellales \\
Steccherinaceae & Pseudohydnum gelatinosum (Scop.) \\
Irpex lacteus (Fr.) Fr.; BPE, DM, L (Fallad 7) & P. Karst.; BPE, C, L (Buenrostro 23, \\
Stereaceae & Guzmán-Dávalos 3873, Zamarripa 106) \\
Stereum complicatum Fr.; BPE, DM, L & Tulostomatales \\
(Guzmán-Dávalos 2243, Vázquez-Morales & Calostoma cinnabarinum Corda; BPE, H \\
26) & (Suárez s.n.) \\
S. frustulatum (Pers.) Fr.; BP, DM, L & Teliomycetes \\
(Gallardo 1) & Uredinales \\
S. gausapatum (Fr.) Fr.; BPE, DM, L & Cronartium conigenum Hedgc. \& N.R. \\
(Gándara 183, García 6, López 10, Marín & Hunt; BPE, C, Fi, W (De Luna 21) \\
34, Vázquez-Morales 18) & *Gymnosporangium clavipes Cooke \& \\
S. hirsutum (Willd.) Pers.; BPE, DM, L & Peck; BPE, Fi (Guzmán-Dávalos 3859) \\
(Gándara 165, Rodríguez 701) & \\
\hline & \\
\hline &
\end{tabular}


